\title{
Interaction of moving D-branes on orbifolds
}

\author{
Faheem Hussain ${ }^{1}$, Roberto Iengo ${ }^{2}$, Carmen Núñez ${ }^{3}$ and Claudio A. Scrucca ${ }^{2}$ \\ 1 International Centre for Theoretical Physics, Trieste, Italy \\ ${ }^{2}$ International School for Advanced Studies and INFN Sezione di Trieste, Trieste, Italy \\ ${ }^{3}$ Instituto de Astronomía y Física del Espacio (CONICET), Buenos Aires, Argentina
}

\begin{abstract}
We use the boundary state formalism to study the interaction of two moving identical D-branes in the Type II superstring theory compactified on orbifolds. By computing the velocity dependence of the amplitude in the limit of large separation we can identify the nature of the different forces between the branes. In particular, in the $Z_{3}$ orbifold case we find a boundary state which is coupled only to the $N=2$ graviton multiplet containing just a graviton and a vector like in the extremal Reissner-Nordström configuration. We also discuss other cases including $T_{4} / Z_{2}$.
\end{abstract}

PACS: $11.25 .-\mathrm{w}$

Keywords: string theory, D-branes 
The non-relativistic dynamics of Dirichlet branes [1], plays an essential role in the understanding of string theory at scales shorter than the Planck length [4,5]. It also provides some evidence [2] for the existence of an underlying eleven dimensional theory [6]. D-brane configurations have also been used to calculate the Bekenstein-Hawking formula for black hole entropy from a microscopic point of view [7].

In this work we use the boundary state technique [8] to compute the interaction between two moving identical D-branes in a TypeIIA or IIB superstring theory compactified down to four dimensions on orbifolds. We then evaluate these amplitudes in the limit of large separation of the branes, that is in the field theory limit. Further by looking at the dependence of these amplitudes on the rapidity we can unambiguously identify the various contributions coming from the exchange of the graviton, vector and scalar fields. The cancellation of the force at zero velocity for the toroidal compactification comes from the exchange of the $N=8$ graviton multiplet containing the graviton, the vectors and the scalars. In the case of the $Z_{3}$ orbifold there is a particular BPS boundary state, corresponding to a D3-brane in TypeIIB, for which the no force condition comes from the cancellation due to the exchange of the $N=2$ graviton multiplet containing the graviton and the graviphoton. Thus, the classical solution corresponding to this configuration would be the Reissner-Nordström black hole. When the D-branes are on the fixed point of the orbifold, this being possible for D0-branes in TypeIIA, there are contributions from both the untwisted and twisted sectors, the latter corresponding to the exchange of vector multiplets containing vectors and scalars. For the case of the orbifold $T_{4} / Z_{2} \times T_{2}$ we find for parallel branes that the BPS cancellation of the force is always like in the toroidal case, apart from when the branes are on fixed points where also additional vector multiplets contribute.

We begin by considering a system of two D-branes in a superstring theory compactified down to four dimensions in the interesting case of the $Z_{3}$ orbifold, which breaks the supersymmetry down to $\mathrm{N}=2$ (the branes will further break it to $\mathrm{N}=1$ ) [9, 10]. In the closed string picture the interaction between two branes is viewed as the exchange of a closed string between two boundary states, geometrically describing a cylinder. In the present work we use 
$\tau$ for the coordinate along the length of the cylinder, $0 \leq \tau \leq l$, and $\sigma$ as the periodic coordinate running from 0 to 1 . We will always consider particle-like D-branes, that is the time coordinate satisfies Neumann boundary conditions, $\partial_{\tau} X^{0}(\tau=0, \sigma)=\partial_{\tau} X^{0}(\tau=l, \sigma)=0$, whereas the three uncompactified space coordinates satisfy Dirichlet boundary conditions, $X^{i}(\tau=0, \sigma)=0, X^{i}(\tau=l, \sigma)=Y^{i}$. The boundary conditions are implemented by suitable boundary states.

To evaluate the interaction between moving branes we calculate the amplitude

$$
\mathcal{A}=\int_{0}^{\infty} d l<B, V, X^{i}=Y^{i}\left|e^{-l H}\right| B, V=0, X^{i}=0>
$$

where we have taken one of the branes to be at rest whereas the other is moving with velocity $V$. The boundary states in position space are given in terms of the momentum states as

$$
\begin{gathered}
\left|B, V=0, X^{i}=0>=\int \frac{d^{3} k}{(2 \pi)^{3}} e^{i k \cdot(X=0)}\right| B, V=0, k> \\
\left|B, V, X^{i}=Y^{i}>=\int \frac{d^{3} q}{(2 \pi)^{3}} e^{i q \cdot Y}\right| B, V, q>.
\end{gathered}
$$

In principle these should contain sums over the discrete compactified momenta $p_{n}$ (they are zero for Neumann boundary conditions in the corresponding directions whereas for Dirichlet compactified directions one has a wave function like for the uncompactified case). Since later we will be interested in the field theory limit, $l \rightarrow \infty$, and since each internal momentum $p_{n}$ will be weighted by a factor $e^{-l p_{n}^{2}}$ from the compact Hamiltonian, only the $p_{n}=0$ term of the sum will be relevant (similarly we ignore the winding states). Thus, the momentum content of the boundary state at rest will be effectively $\left(k^{0}=0, \vec{k}, p_{n}=0\right)$.

We consider the case of two branes, one of them moving along one of the uncompactified space directions, say the $X^{1}$ direction. We make pairs of fields, $X^{A}=X^{0}+X^{1}$ and $X^{B}=$ $X^{0}-X^{1}$, and pair the $X^{2}$ and $X^{3}$ into the complex fields $X^{2} \pm i X^{3}$. The compact directions are treated in terms of three pairs of complex cordinates [9]. The net effect is as if the $b-c$ ghosts cancel the contribution of the pair of coordinates, $X^{2}$ and $X^{3}$, orthogonal to the boost. Similarly the $\beta-\gamma$ ghost contribution cancels the contribution from the fermionic pair $\psi^{3}$ and $\psi^{4}$ for each spin structure. 
In order to get the spacetime contribution to the boundary state of the D-brane moving with constant velocity $\mathrm{V}$, let us consider the boost [1]] $\left|B_{v}>=e^{-i v^{j} J_{j}^{0}}\right| B>$, where $V=$ $\tanh v,\left(v=\left|v^{j}\right|\right)$, is the velocity and $J^{\mu \nu}$ is the Lorentz generator.

The full amplitude is a product of the amplitudes for the bosonic and fermionic coordinates. We first consider the bosonic coordinates. With the standard commutation relations for the bosonic oscillators $\left[a_{n}^{\nu}, a_{-m}^{\nu}\right]=\left[\tilde{a}_{n}^{\nu}, \tilde{a}_{-m}^{\nu}\right]=\eta^{\mu \nu} \delta_{n m}$, the oscillators, $\left(\alpha_{n}, \beta_{n}\right)$, for the $X^{A}$ and $X^{B}$ fields, respectively, are now defined as $\alpha_{n}=a_{n}^{0}+a_{n}^{1}, \beta_{n}=a_{n}^{0}-a_{n}^{1}$, etc., with the commutation relations $\left[\alpha_{n}, \beta_{-m}\right]=\left[\tilde{\alpha}_{n}, \tilde{\beta}_{-m}\right]=-2 \delta_{n m}$, and the other commutators being zero.

The Neumann boundary conditions for the time and Dirichlet for the space coordinates translate into

$$
\left(\alpha_{n}+\tilde{\beta}_{-n}\right)\left|B>=0, \quad\left(\beta_{n}+\tilde{\alpha}_{-n}\right)\right| B>=0 .
$$

Here $\mid B>$ is the unboosted bosonic spacetime part of the boundary state. Under a Lorentz boost in the 1 direction the oscillators transform as

$$
\alpha_{n} \rightarrow e^{-v} \alpha_{n} \quad \beta_{n} \rightarrow e^{v} \beta_{n}
$$

and similarly for the $\tilde{\alpha}_{n}, \tilde{\beta}_{n}$. The bosonic spacetime part of the boundary state of the moving brane is then

$$
\left|B_{v}>=\exp \sum_{n>0}\left\{\frac{1}{2}\left(e^{-2 v} \alpha_{-n} \tilde{\alpha}_{-n}+e^{2 v} \beta_{-n} \tilde{\beta}_{-n}\right)+a_{-n}^{T} . \tilde{a}_{-n}^{T}\right\}\right| 0>,
$$

where $a_{n}^{T}$ denote the oscillators of the directions orthogonal to the motion of the brane. The momentum content of the boosted state will be $q^{0}=\sinh (v) q^{1}, \vec{q}=\left(\cosh (v) q^{1}, q_{\perp}\right)$ Therefore from momentum conservation in eq. (四) we will get $q^{1}=k^{1}=0, q_{\perp}=k_{\perp}$ and thus we get the amplitude at fixed impact parameter $Y_{\perp}$ as

$$
\mathcal{A}=\int d^{3} k_{\perp} e^{i k_{\perp} Y_{\perp}} \int_{0}^{\infty} d l M\left(l, k_{\perp}\right)
$$

In the following we write $M=Z_{B} Z_{F}$ where $Z_{B, F}$ are the bosonic, fermionic contributions. We start by computing the matrix element representing the bosonic spacetime coordinates contribution to the amplitude 


$$
Z_{B}=<B_{v}\left|e^{-l H}\right| B>
$$

where $H$ is the usual closed string Hamiltonian.

The oscillator part of the bosonic spacetime contribution is computed to be [1]

$$
\prod_{n=1}^{\infty} \frac{1}{\left(1-e^{-2 v} e^{-4 \pi l n}\right)\left(1-e^{2 v} e^{-4 \pi l n}\right)}=\frac{2 f\left(q^{2}\right) q^{1 / 4} i \sinh v}{\vartheta_{1}\left(\frac{v i v}{\pi} \mid 2 i l\right)},
$$

where $f\left(q^{2}\right)=\prod_{n=1}^{\infty}\left(1-q^{2 n}\right)$ with $q=e^{-2 \pi l}$.

Let us now introduce the standard $Z_{3}$ orbifold [9], that is compactifying the $\mu=$ $4,5,6,7,8,9$ coordinates on a 6 -torus and identifying points which are equivalent under $g_{a}=e^{2 \pi i z_{a}}$ rotations on pairs of them, with $z_{4,5}=z_{6,7}= \pm 1 / 3$ and $z_{8,9}=-z_{4,5}-z_{6,7}$ for the pairs $X^{4,5}=X^{4}+i X^{5}, X^{6,7}=X^{6}+i X^{7}, X^{8,9}=X^{8}+i X^{9}$ respectively. The request of the same Neumann or Dirichlet b.c.s for both members of a pair is, with $\beta_{n}^{4,5}=a_{n}^{4}+i a_{n}^{5}$, $\beta_{n}^{4,5 *}=a_{n}^{4}-i a_{n}^{5}$ etc.

$$
\left(\beta_{n}^{a} \pm \tilde{\beta}_{-n}^{a}\right)\left|B>=0 \quad\left(\beta_{n}^{a *} \pm \tilde{\beta}_{-n}^{a *}\right)\right| B>=0
$$

and the corresponding boundary state is

$$
\left|B>=\prod_{a} \exp \mp \frac{1}{2} \sum_{n \geq 1}\left(\beta_{-n}^{a} \tilde{\beta}_{-n}^{a *}+\beta_{-n}^{a *} \tilde{\beta}_{-n}^{a}\right)\right| 0>.
$$

This is the same as for the torus compactification, except that in the orbifold case there could be a twist in the $\sigma$-direction giving noninteger moding, which we discuss in a while. However the presence of the orbifold opens new possibilities for BPS states. In fact, let us consider D3-branes in TypeIIB theory with Neumann b.c.s for $X^{i}$ and Dirichlet b.c.s for $X^{i+1}(i=4,6,8)$ at both ends $\tau=0, l$. That is

$$
\left(\beta_{n}^{a}+\tilde{\beta}_{-n}^{a *}\right)\left|B>=0 \quad\left(\beta_{n}^{a *}+\tilde{\beta}_{-n}^{a}\right)\right| B>=0
$$

and the corresponding boundary state is

$$
\left|B>=\prod_{a} \exp -\frac{1}{2} \sum_{n \geq 1}\left(\beta_{-n}^{a} \tilde{\beta}_{-n}^{a}+\beta_{-n}^{a *} \tilde{\beta}_{-n}^{a *}\right)\right| 0>.
$$

The physical boundary state, which is required to be $Z_{3}$ invariant, is 


$$
\mid B_{\text {phys }}>=\frac{1}{3}\left(|B, 1>+| B, g>+\mid B, g^{2}>\right)
$$

where we have introduced the "twisted boundary state", $\left(g^{a} \beta_{n}^{a}+g^{a *} \tilde{\beta}_{-n}^{a *}\right) \mid B, g>=0$ :

$$
\left|B, g>=\prod_{a} \exp -\frac{1}{2} \sum_{n \geq 1}\left(\left(g^{a}\right)^{2} \beta_{-n}^{a} \tilde{\beta}_{-n}^{a}+\left(g^{a *}\right)^{2} \beta_{-n}^{a *} \tilde{\beta}_{-n}^{a *}\right)\right| 0>.
$$

Since $g^{2}$ is generically an element of $Z_{3}$ we will write, in the following, $g \beta \tilde{\beta}$ for $g^{2} \beta \tilde{\beta}$. One gets for a pair of coordinates $\left(g_{a}^{*} g_{a}^{\prime}=e^{2 \pi i z_{a}}\right)$

$$
<B_{a}, g_{a}\left|e^{-l H}\right| B_{a}, g_{a}^{\prime}>=\prod_{n=1}^{\infty}\left|\frac{1}{1-g_{a}^{*} g_{a}^{\prime} e^{-4 \pi l n}}\right|^{2}=\frac{2 f\left(q^{2}\right) q^{1 / 4} \sin \left(\pi z_{a}\right)}{\vartheta_{1}\left(z_{a} \mid 2 i l\right)}
$$

Taking now into account all the contributions from the compactified directions as well as the spacetime sector and the normal ordering term from the Hamiltonian $\left(q^{-2 / 3}\right)$, it is seen that the oscillator part of the bosonic amplitude is

$$
Z\left(g, g^{\prime}\right)_{B}=i\left[2 f\left(q^{2}\right)\right]^{4} \frac{q^{1 / 3} \sinh (v)}{\vartheta_{1}\left(\frac{i v}{\pi} \mid 2 i l\right)} \prod_{a} \frac{\sin \left(\pi z_{a}\right)}{\vartheta_{1}\left(z_{a} \mid 2 i l\right)} .
$$

On the orbifold $\sigma$-twisted sectors are also possible. We will be concerned with this sector only in the case when the branes are on an orbifold fixed point, since only then the twisted closed string is shrinkable to zero. Thus we consider this sector only for Dirichlet b.c.s on every compact coordinate (thus for D0-branes in TypeIIA) and the corresponding boundary state is the one of eq. (10). In this sector the pairs of fields in the compactified directions may be diagonalized [9] such that

$$
X^{a, b}(\sigma+1)=e^{2 \pi i z_{a}} X^{a, b}(\sigma), \quad X^{* a, b}(\sigma+1)=e^{-2 \pi i z_{a}} X^{* a, b}(\sigma),
$$

with $(a, b)=(4,5),(6,7),(8,9)$. This leads to fractional moding of the oscillators. The oscillator part of the bosonic amplitude for a pair of cordinates $X^{a, b}$ becomes

$$
<B_{a}\left|e^{-l H}\right| B_{a}>=\prod_{n=1}^{\infty}\left(1-e^{-4 \pi l\left(n-\frac{1}{3}\right)}\right)^{-1}\left(1-e^{-4 \pi l\left(n-\frac{2}{3}\right)}\right)^{-1} .
$$

Combining with the spacetime part and converting to Jacobi theta functions we get the full bosonic amplitude to be 


$$
Z_{B}(\sigma-t w i s t e d)=2\left[f\left(q^{2}\right)\right]^{4} \frac{\sinh (v)}{\vartheta_{1}\left(\frac{i v}{\pi} \mid 2 i l\right)} \vartheta_{1}(-2 i l / 3 \mid 2 i l)^{-3} .
$$

Now we consider the fermionic modes' contribution. Again here we combine the fermionic coordinates $\psi^{0}$ and $\psi^{1}$ into a pair of coordinates $\psi^{A}=\psi^{0}+\psi^{1}$ and $\psi^{B}=\psi^{0}-\psi^{1}$. The oscillators satisfy the anti-commutation relations $\left\{\psi_{m}^{A}, \psi_{n}^{B}\right\}=\left\{\tilde{\psi}_{m}^{A}, \tilde{\psi}_{n}^{B}\right\}=-2 \delta_{m n}$ with appropriate half-integer or integer moding for the NS-NS and RR cases. The other directions are combined into complex pairs $(2,3),(4,5),(6,7),(8,9)$ as before. With the appropriate normalisation the spacetime modes' b.c.s for the D-brane at rest are given by [8] (Neumann for time and Dirichlet for space)

$$
\left(\psi_{n}^{0}+i \eta \tilde{\psi}_{-n}^{0}\right)\left|B, \eta>=0, \quad\left(\psi_{n}^{i}-i \eta \tilde{\psi}_{-n}^{i}\right)\right| B, \eta>=0
$$

Here $\eta= \pm 1$ has been introduced to deal with the GSO projection. For the longitudinal coordinates these can be rewritten as $\left(\psi_{n}^{A}+i \eta \tilde{\psi}_{-n}^{B}\right)\left|B>_{F}=0,\left(\psi_{n}^{B}+i \eta \tilde{\psi}_{-n}^{A}\right)\right| B>_{F}=0$

Now to construct the moving boundary state we note that under the boost in the $X^{1}$ direction the fields $\psi^{A}$ and $\psi^{B}$ transform like the bosonic coordinates $\psi^{A} \rightarrow e^{-v} \psi^{A}, \psi^{B} \rightarrow$ $e^{v} \psi^{B}$. Thus the fermionic spacetime part of the boundary state of the moving brane is found to be

$$
\mid B_{v}, \eta>=\exp \sum_{m>0}\left\{\frac{i \eta}{2}\left(e^{-2 v} \psi_{-m}^{A} \tilde{\psi}_{-m}^{A}+e^{2 v} \psi_{-m}^{B} \tilde{\psi}_{-m}^{B}\right)-i \eta \psi_{-m}^{T} \tilde{\psi}_{-m}^{T}\right\} .
$$

The zero modes of the four uncompactified coordinates $\psi^{\mu}$, with $\mu=0,1,2,3$ for the R-R state can be identified with the $\gamma$-matrices $\gamma^{\mu}=i \sqrt{2} \psi_{0}^{\mu}$ and $\tilde{\gamma}^{\mu}=i \sqrt{2} \tilde{\psi}_{0}^{\mu}$, with $\left\{\gamma^{\mu}, \gamma^{\nu}\right\}=$ $-2 \eta^{\mu \nu}$, which act on a subspace which is a direct product of two spinor spaces. We define $a=\left(\gamma^{0}+\gamma^{1}\right) / 2, a^{*}=\left(\gamma^{0}-\gamma^{1}\right) / 2$ and $b=\left(-i \gamma^{2}+\gamma^{3}\right) / 2, b^{*}=\left(-i \gamma^{2}-\gamma^{3}\right) / 2$ and similarly for $\tilde{a}, \tilde{b}$ and a vacuum by $a|0>=b| 0>=\tilde{a}\left|\tilde{0}>=\tilde{b}^{*}\right| \tilde{0}>=0$. Under the boost we have the transformations $a \rightarrow e^{-v} a, a^{*} \rightarrow e^{v} a^{*}$ leading to the boosted boundary state $\mid B_{v}, \eta>=$ $e^{v \gamma^{0} \gamma^{1} / 2} \mid B, v=0>$, giving for the RR zero mode part of the moving boundary state

$$
\left|B_{v}, \eta>=\frac{e^{-v}}{\sqrt{2}} e^{-i \eta\left(e^{2 v} a^{*} \tilde{a}^{*}-b^{*} \tilde{b}\right)}\right| 0>\otimes \mid \tilde{0}>
$$

The GSO projected state in both the NS-NS and RR sectors is 


$$
\mid \mathcal{B}>_{N S, R}=\frac{1}{2}\left\{\left|B, \eta>_{N S, R}-\right| B,-\eta>_{N S, R}\right\} .
$$

We now compute the matrix element $\left\langle\mathcal{B}_{v}\left|e^{-l H}\right| \mathcal{B}\right\rangle_{F}$ (with the appropriate Hamiltonians for the NS-NS and RR sectors [10]) for the fermions. Thus the spacetime part of the fermionic amplitude turns out to be

$$
<B_{v}, \eta\left|e^{-l H}\right| B, \eta^{\prime}>=\prod_{n>0}\left(1 \pm e^{2 v} q^{2 n}\right)\left(1 \pm e^{-2 v} q^{2 n}\right) \cdot Z_{0}( \pm),
$$

where $n$ is an integer or half integer for NS-NS or RR, $\eta \eta^{\prime}= \pm$ for the two possible cases of the GSO projection and $Z_{0}( \pm)=1$ for NS-NS, $Z_{0}(+)=2 \cosh (v)$ and $Z_{0}(-)=0$ for RR. The $\psi^{2,3}$ contribution is cancelled by the $\beta-\gamma$ ghosts, but in the $\mathrm{RR} \eta \eta^{\prime}=-1$ (odd spin structure) case there remains the zero mode of this pair giving zero.

For the compactified coordinates one again combines the fermions into pairs $\chi_{n}^{4,5}=$ $\psi_{n}^{4}+i \psi_{n}^{5}$, etc. Let us describe the fermionic part of the previously introduced twisted boundary state (14) (mixed Neumann/Dirichlet b.c.s for each pair). The condition for the twisted boundary state is now

$$
\left(g^{a} \chi_{n}^{a}+i g^{a *} \eta \tilde{\chi}_{-n}^{a *}\right) \mid B, g_{a}, \eta>=0,
$$

for each pair of coordinates and the state satisfying this condition is (for generic $a$ and $g$ )

$$
\mid B, g, \eta>=\exp \left\{\frac{i \eta}{2} \sum_{n>0}\left(g \chi_{-n} \tilde{\chi}_{-n}+g^{*} \chi_{-n}^{*} \tilde{\chi}_{-n}^{*}\right\} \mid 0>.\right.
$$

Using these states we find for a pair of fermionic coordinates in the compactified space, $\sigma$-untwisted closed string sector, that

$$
<B, g, \eta\left|e^{-l H}\right| B, g^{\prime} \eta^{\prime}>=\prod_{n>0}\left|1 \pm e^{2 \pi i z_{a}} q^{2 n}\right|^{2} \cdot Z_{0}^{c}( \pm) .
$$

Now $Z_{0}^{c}( \pm)=1$ for the NS-NS sector, $Z_{0}^{c}(+)=2 \cos \pi z_{a}$ and $Z_{0}^{c}(-)=2 i \sin \pi z_{a}$ for the RR sector.

Putting everything together, taking into account all the compactified directions as well as the spacetime contribution and the normal ordering terms for both the NS-NS sector 
and the RR sector we find, for the twisted boundary state ( $\sigma$-untwisted sector), that the fermionic amplitude is, in terms of Jacobi theta functions,

$$
\begin{aligned}
& <\mathcal{B}_{v}, g\left|e^{l H}\right| \mathcal{B}, g^{\prime}>_{F}=\frac{1}{q^{1 / 3} f\left(q^{2}\right)^{4}} \times \\
& \left\{\vartheta_{2}\left(\frac{i v}{\pi} \mid 2 i l\right) \prod_{a} \vartheta_{2}\left(z_{a} \mid 2 i l\right)-\vartheta_{3}\left(\frac{i v}{\pi} \mid 2 i l\right) \prod_{a} \vartheta_{3}\left(z_{a} \mid 2 i l\right)+\vartheta_{4}\left(\frac{i v}{\pi} \mid 2 i l\right) \prod_{a} \vartheta_{4}\left(z_{a} \mid 2 i l\right)\right\} .
\end{aligned}
$$

In making the algebraic sum of the RR and NS-NS sectors, we take the same signs which hold for the partition function on the torus. The first term is the contribution from the RR sector (even spin structure) whereas the second and the third are from the two NS-NS GSO projections. Observe that the compactified parts of eqs. (16) and (28) agree with the $\vartheta$-function expressions on the torus found in ref. [9]. This is consistent with viewing the cylinder as half of a torus, and also the $\langle\psi \psi\rangle$ correlators computed with the b.c. (25) would agree with those on the torus with Minahan's twist [9] in the $\tau$-direction. Since the $Z_{3}$ invariant, physical boundary state is given by the linear combination of eq. (13), one has still to sum the product of the bosonic (16) and fermionic (28) amplitudes over the three possibilities for $g$ and $g^{\prime}$ (actually only three possibilities for $g^{-1} g^{\prime}$ are distinct).

On using the Riemann identity it is easy to see that the amplitude (28) behaves for small velocities as $V^{2}$, if $g \neq g^{\prime}$ and as $V^{4}$ if $g=g^{\prime}$. In the limit $l \rightarrow \infty$ (where the bosonic part (16) is $z_{a}$ independent) the leading behaviour of this amplitude is proportional to

$$
\left\{4 \cosh (v) \prod_{a} \cos \left(\pi z_{a}\right)-\left(\cosh 2 v+\sum_{a} \cos \left(2 \pi z_{a}\right)\right\}\right.
$$

We observe here that the first term with $\cosh (v)$ is the contribution of the $\mathrm{RR}$ vector whereas the rest are the contributions from NS-NS exchange. We note that the amplitude (28) vanishes at $v=0$ for all the twists $\left(1, g, g^{2}\right)$ individually.

Just as for the bosonic amplitude, when the position of the brane is on the fixed point of the orbifold here we also have to include the closed string $\sigma$-twisted sectors. Here too the oscillator moding is modified from the usual integer and half-integer in the RR and NS-NS sectors [10]. By grouping the coordinates into pairs we find for each pair of compactified coordinates in the NS-NS sector that 


$$
<B, \eta\left|e^{-l H}\right| B, \eta^{\prime}>_{N S}=\prod_{n=1}^{\infty}\left[1 \pm e^{-4 \pi l\left(n-\frac{5}{6}\right)}\right]\left[1 \pm e^{-4 \pi l\left(n-\frac{1}{6}\right)}\right]
$$

For the RR sector (in this twisted sector there are no zero modes) we have here

$$
<B, \eta\left|e^{-l H}\right| B, \eta^{\prime}>_{R}=\prod_{n=1}^{\infty}\left[1 \pm e^{-4 \pi l\left(n-\frac{1}{3}\right)}\right]\left[1 \pm e^{-4 \pi l\left(n-\frac{2}{3}\right)}\right]
$$

The net result in the twisted sector is that the full fermionic amplitude, including the spacetime sector and the appropriate normal ordering contributions, can now be written in terms of Jacobi theta functions as

$$
\begin{aligned}
& <\mathcal{B}_{v}\left|e^{-l H}\right| \mathcal{B},>_{F}=f\left(q^{2}\right)^{-4}\left\{\vartheta_{2}\left(\frac{i v}{\pi} \mid 2 i l\right) \vartheta_{2}(-2 i l / 3 \mid 2 i l)^{3}\right. \\
& \left.-\vartheta_{3}\left(\frac{i v}{\pi} \mid 2 i l\right) \vartheta_{3}(-2 i l / 3 \mid 2 i l)^{3}-\vartheta_{4}\left(\frac{i v}{\pi} \mid 2 i l\right) \vartheta_{4}(-2 i l / 3 \mid 2 i l)^{3}\right\} .
\end{aligned}
$$

Recall that in the twisted sector for the $Z_{3}$ orbifold there has to be a relative positive sign between the two NS sectors because of invariance under the modular transformation $\tau \rightarrow \tau+3$. At low velocities this amplitude goes like $V^{2}$.

Let us now compare the large distance interactions of the two moving branes found from the string formalism with the field theory results. At large distances we look for the Feynman graphs representing the exchange of massless particles. We can have the exchange either of scalar, or vector or graviton. The scalar and the graviton give attraction while the vector gives repulsion, since we consider two branes of the same nature. The net result for zero velocity is zero, since the branes are BPS states, and this is what is obtained from the Riemann identity in the string formalism [12]. But when the velocity is different from zero, the various contributions are unbalanced. By comparing the velocity dependence with what we get from Feynman graphs we can tell which kind of particles are actually coupled to the branes, in various compactification cases.

We treat the branes as spinless particles of mass and charge equal to 1 . The exchange of a scalar gives then

$$
\mathcal{S}=\frac{1}{k_{\perp}^{2}}
$$


where $k$ is the momentum transfer between the two branes. In the so-called eikonal approximation in which the branes go straight (which is the standard setting, which we follow, for describing the branes' interaction at nonsmall distances), $k$ has only space components, and it is orthogonal to $\vec{V}$.

The vector is coupled to the current, which in the eikonal approximation is proportional to the momentum, $J^{\mu}(V) \equiv(\cosh (v), \sinh (v))$. Note that $J^{\mu} k_{\mu}=0$. Taking one of the branes at rest, the vector exchange is

$$
\mathcal{V}=J^{\mu}(V) J_{\mu}(0) \frac{1}{k_{\perp}^{2}}=-\frac{\cosh (v)}{k_{\perp}^{2}}
$$

The graviton is coupled to the brane's energy-momentum tensor $T^{\mu \nu}=J^{\mu} J^{\nu}$. Therefore the graviton exchange in d-dimensions is

$$
\mathcal{G}=2\left(T^{\mu \nu}(V)-\frac{\eta^{\mu \nu}}{d-2} T^{\rho \sigma}(V) \eta_{\rho \sigma}\right) T_{\mu \nu}(0) \frac{1}{k_{\perp}^{2}}=\frac{\cosh (2 v)+\frac{d-4}{d-2}}{k_{\perp}^{2}} .
$$

Thus we see that we can tell the nature of the various contributions to the branes' interaction by looking at the rapidity dependence of the $l \rightarrow \infty$ limit of the amplitude $Z_{B} \cdot Z_{F}$ (after removing the $\sinh (v)$ factor in the denominator, the eikonal amplitude being the relativistically invariant interaction divided by that factor).

We now discuss various cases.

1.) Toroidal compactification. The boundary states can be of the form of eqs. (10) or (12) and in any case one gets eqs. (28) and (29) with $z_{a}=0$. Thus in the field theory limit the amplitude is proportional to

$$
4 \cosh (v)-\cosh (2 v)-3
$$

Take in particular the case of two D0-branes. If we consider eq. (36) as the result for the ten dimensional uncompactified case we write it as $4 \cosh (v)-\left(\cosh (2 v)+\frac{3}{4}\right)-\frac{9}{4}$. The first term is the contribution from the exchange of the $\mathrm{RR}$ vector whereas the second term is from the exchange of the graviton in 10 dimensions (as is seen from the field theory calculation above) and the last is the scalar exchange contribution. We have normalised to the graviton 
exchange. Our D0-brane in 10 dimensions is like the classical 0-brane solution [13] of the bosonic part of the Type IIA 10 dimensional supergravity action.

If we now view eq.(36) from the point of view of toroidal compactification, with no SUSY breaking, in four dimensions the graviton exchange gives the contribution $\cosh (2 v)$. Our toroidal compactification corresponds to Stelle's vertical reduction of the 10 dimensional 0-brane solution to four dimensions [13]. In this case the relations between the masses, the electric charge and the scalar coupling of the 0-brane are $Q^{2}=4 M^{2}$ and $a^{2}=3 M^{2}$, precisely the relations that we have obtained. Further, in both these cases, uncompactified and toroidally compactified, the force between the branes goes to zero like $V^{4}$ as expected as there is no supersymmetry breaking. Following the work of Pollard [14] we also see that the D0-branes in this case are extremal Dobiasch-Maison blackholes [15]. This extremal blackhole has zero horizon radius and zero horizon area.

2.) Orbifold compactification, $\sigma$-untwisted sector. For the interaction of two branes with the same Neumann or Dirichlet b.c.s for both members of pairs of compactified coordinates (thus for TypeIIA) we have the same result as for toroidal compactification. If instead we take the two branes to be in the mixed Neumann/Dirichlet configuration corresponding to the boundary state of eq. (13) (thus for D3-branes in TypeIIB), we find, by summing eq. (29) over the allowed $z_{a}$ 's, that at large brane separation the amplitude is proportional to

$$
\cosh (v)-\cosh (2 v)
$$

Thus, these two branes interact through the exchange of the RR vector and the universal graviton with no scalar exchange. In terms of the $\mathrm{N}=2 \mathrm{SUSY}$ theory these systems couple only to the graviton and its $\mathrm{N}=2$ partner, the graviphoton. In this case the amplitude behaves like $V^{2}$ for small velocities. From the pattern of cancellation [14 we see that these branes correspond to classical extremal Reissner-Nordström blackholes.

3.) Orbifold compactification, $\sigma$-twisted sector. In this case the $l \rightarrow \infty$ limit of eq.(32) gives

$$
\cosh (v)-1
$$


When the branes are on the fixed point (Dirichlet b.c.s for every compactified coordinate, TypeIIA) they interact through the interchange of all three fields, because we have contributions from both untwisted (giving in this case the toroidal result) and twisted sectors. Here we have extra vectors as well as scalars and the force between the branes falls off as $V^{2}$.

It is now an easy exercise to repeat the above calculation for the $T_{4} / Z_{2} \times T_{2}$ orbifold which reduces the supersymmetry to $\mathrm{N}=4$ rather than $\mathrm{N}=2$. Here in the $\sigma$-untwisted sector, we can also construct the mixed Neumann-Dirichlet twisted boundary state for the first two coordinate pairs (in this case it could be done for both TypeIIA and TypeIIB). However since in this case the twists on the two pairs of coordinates $X^{4}, X^{5}$ and $X^{6}, X^{7}$ are $g_{a}=\exp \left(2 \pi i z_{a}\right)$ with $z_{4,5}=-z_{6,7}=1 / 2$, and thus $g^{2} \beta \tilde{\beta}=\beta \tilde{\beta}$, then in the notation of eqs. (13),(14) $|B, g>=| B, 1>$ and $\left|B_{\text {phys }}>=\right| B, 1>$. Therefore the interaction between two parallel branes in this case behaves like the toroidal compactification, the force falling off like $V^{4}[16]$.

For the $\sigma$-twisted sector the fermionic partition function is proportional to

$$
\begin{aligned}
& \left\{\vartheta_{2}\left(\frac{i v}{\pi} \mid 2 i l\right) \vartheta_{2}(0 \mid 2 i l) \vartheta_{2}(-i l \mid 2 i l)^{2}\right. \\
& \left.-\vartheta_{3}\left(\frac{i v}{\pi} \mid 2 i l\right) \vartheta_{3}(0 \mid 2 i l) \vartheta_{3}(-i l \mid 2 i l)^{2}-\vartheta_{4}\left(\frac{i v}{\pi} \mid 2 i l\right) \vartheta_{4}(0 \mid 2 i l) \vartheta_{4}(-i l \mid 2 i l)^{2}\right\}
\end{aligned}
$$

Again in the twisted sector for large separation we find that the amplitude is proportional to $\cosh (v)-1$ and the force falls off as $V^{2}$.

Acknowledgements. The authors would like to thank E. Gava and K.S. Narain for very valuable discussions. C.A.S. also thanks F. Morales and M. Serone for useful exchange of ideas. C.N. would like to thank ICTP for hospitality during the completion of this work, which was done in the frame of the Associate Membership Programme of the ICTP. R.I. and C.A.S. acknowledge partial support from EEC contract ERBFMRXCT96-0045. 


\section{REFERENCES}

[1] C. Bachas, Phys. Lett. 374B (1996) 37;

C. Bachas, (Half) a lecture on D-branes, Workshop on Gauge Theories, Applied Supersymmetry and Quantum Gravity, Imperial College, London (July 1996).

[2] G. Lifschytz, "Comparing D-branes to Black-branes", hep-th/9604156

[3] C. Bachas and M. Porrati, Phys. Lett. B296 (1992) 77

[4] D. Kabat and P. Pouliot, "A Comment on Zero-Brane Quantum Mechanics", hepth/9603127;

U. H. Danielsson, G. Ferretti and B. Sundborg, "D-Particle Dynamics and Bound States", hep-th/9603081

[5] S. H. Shenker, "Another length scale in String Theory?", hep-th/9509132;

M. Douglas, D. Kabat, P. Pouliot and S. Shenker, "D-branes and Short Distances in String Theory", hep-th/9608024

[6] E. Witten, Nucl. Phys. B443 (1995) 85

J. Schwarz, Phys. Lett. B360 (1995) 13; B367 (1996) 97

[7] A. Strominger and C. Vafa, "Microscopic origin of the Bekenstein-Hawking entropy", hep-th/9601029;

C.G. Callan,Jr. and J.M. Maldacena, "D-brane approach to Black Hole Quantum Mechanics", hep-th/9602043;

G. Horowitz and A. Strominger, "Counting States of Near-Extremal Black Holes", hepth/9602051;

C.V. Johnson, R.R. Khuri and R.C. Myers, "Entropy of 4 D Extremal Black Holes", hep-th/9603061

[8] J. Polchinski and Y. Cai, Nucl. Phys. B296 (1988) 91

[9] J. A. Minahan, Nucl. Phys. B298 (1988) 36 
[10] F. Hussain, R. Iengo and C. Núñez, Axion production from gravitons off interacting 0-branes, hep-th/9701143, IC/97/1, SISSAREF-3/97/EP, to appear in Nucl. Phys. B

[11] M. Billó, P. Di Vecchia and D. Cangemi, "Boundary states for moving D-branes", hepth/9701190, NBI-HE-97-05, NORDITA 97/7P

[12] J. Polchinski, Phys. Rev Lett. 75 (1995) 4724

[13] K.S. Stelle, Lectures on supergravity p-branes, ICTP Summer School in High Energy Physics and Cosmology, Trieste, June 10-26, 1996. hep-th/9701088

[14] D. Pollard, J. Phys.A 16 (1983) 565

[15] P. Dobaisch and D. Maison, GRG 14 (1982) 393

[16] M.R. Douglas, H. Ooguri and S.H. Shenker, "Issues in M(atrix) Theory Compactification", hep-th/9702203 